\title{
Valores e Confiança Organizacionais: Fatores Determinantes nos Comportamentos de Civismo Organizacional
}

\author{
Fabiana Rocha Dias ${ }^{1}$ \\ Faculdades Integradas Pitágoras, Uberlândia, MG, Brasil \\ Áurea de Fátima Oliveira \\ Instituto de Psicologia da Universidade Federal de Uberlândia, Uberlândia, MG, Brasil
}

\begin{abstract}
Resumo
Este estudo teve como objetivo verificar o impacto de valores e confiança organizacionais sobre comportamentos de civismo. Participaram do estudo 206 trabalhadores, de diversas organizações, que responderam a instrumentos fidedignos e válidos referentes a cada construto: Inventário de Perfis de Valores Organizacionais, Inventário de Confiança do Empregado na Organização e Escala de Civismo nas Organizações. Os coeficientes de confiabilidade das escalas para a amostra do estudo foram superiores a 0,70, permitindo a inclusão de todas as variáveis nas análises de regressão múltipla padrão. As variáveis dependentes foram os fatores de civismo organizacional enquanto as independentes foram os valores organizacionais e os fatores de confiança. Os resultados mostraram que confiança na ética e na competência organizacional e os valores organizacionais conformidade, autonomia e coletividade foram preditores das dimensões de civismo organizacional. A conclusão do estudo indica a importância dessas variáveis na emissão de comportamentos de civismo organizacional, sugerindo, ainda, a realização de estudos abrangendo outras amostras bem como a inclusão de novas variáveis em modelos de investigação futuros.
\end{abstract}

Palavras-chave: Civismo organizacional, valores organizacionais, confiança.

\section{Organizational Values and Trust: Determining Factors in Organizational Civism Behaviors}

\begin{abstract}
This study aimed to verify the impact of organizational values and trust on civism behaviors. Participants were 206 employees from several organizations, who responded to reliable and valid instruments related to each construct: Inventory of Organizational Values Profile, Inventory Employee Trust in the Organization and Civism Scale in Organizations. The reliability coefficients of the scales for the study sample were higher than .70, thus they allowed the inclusion of all the variables in the standard multiple regression analysis. The factors of organizational civism were the dependent variables, whereas the organizational values and the factors of trust composed the independent variables. The results demonstrated the trust in ethics and organizational competence and the organizational values of conformity, autonomy and collectivity were predictors of the organizational civism dimensions. The conclusion of the study indicates the importance of those variables in the emission of organizational
\end{abstract}

Endereço para correspondência: Rua Paraná, 812, Bairro Brasil, Uberlândia, MG, Brasil 38400-654. E-mail: fabianarochadias@gmail.com 
Civism behaviors, while also suggesting the performance of studies with other samples and the inclusion of new variables for future investigations.

Keywords: Civism behaviours, organizational values, trust.

\section{Los Valores Organizacionales y de Confianza: Factores Determinantes en el Comportamiento de la Civilidad Organizacional}

\section{Resumen}

Este estudio tuvo como objetivo verificar el impacto de los valores y de confianza en la organización en los comportamientos de civismo. Participaron del estudio 206 trabajadores, de varias organizaciones, que respondieron a instrumentos fidedignos y validos referentes a cada constructo: Inventario de los valores de la organización Perfil, la confianza de los empleados de inventario en la Escala de Organización y civilidad en las Organizaciones. Los coeficientes de confiabilidad de las escalas para la muestra del estudio fueron superiores a .70, permitiendo la inclusión de todas las variables en los análisis de regresión múltiple estándar. Las variables dependientes fueron los factores de civismo organizacional mientras las independientes fueron los valores organizacionales y los factores de confianza. Los resultados mostraron que la confianza en la ética y en la competencia organizacional sumada a los valores organizacionales de conformidad, autonomía y colectividad predijeron las dimensiones de civismo organizacional. La conclusión del estudio enseña la importancia de estas variables en la emisión de comportamientos de civismo organizacional, sugiriendo, sin embargo, la realización de estudios abarcando otras muestras así como la inclusión de nuevas variables en modelos futuros de investigación.

Palabras clave: Civismo organizacional, valores organizacionales, confianza.

Comportamentos de cidadania organizacional tem sido estudados desde 1980, embora tais comportamentos não sejam explicitamente reconhecidos pelo sistema formal de recompensa (Cavazotte, Hartman, \& Bahiense, 2013). Tal interesse pode ser atribuído à sua importância no que se refere ao alcance das metas, contribuindo para a efetividade organizacional. Rauf (2014) descreve dentre os vários benefícios a satisfação dos clientes, a qualidade e a quantidade de produtos e serviços e o retorno nos percentuais de venda.

Comportamentos de Cidadania Organizacional referem-se a contribuições informais que as pessoas podem ofertar, ou sonegar, livres da possibilidade de sanções ou incentivos formais (Organ, 1990). Portanto, emergem independentemente de regras ou de retribuições que o indivíduo possa receber; isto é, são comportamentos além do que é determinado pelas organizações, e o trabalhador poderá cooperar de modo espontâneo com sua organização de trabalho e cole- gas (Bastos, Siqueira, \& Gomes, 2014; Siqueira, 1995).

Bolino, Klotz, Turnley, e Harvey (2013) consideram que este comportamento se ajusta ao Comportamento Organizacional Positivo, pois refere-se aos atos dos empregados que apoiam o ambiente social e psicológico no contexto de trabalho em que se inserem. Comportamentos de Cidadania podem abranger ajuda pessoal a outras pessoas, assim como ajuda direcionada à organização (Cavazotte et al., 2013).

Uma vez que estes comportamentos contribuem para os resultados organizacionais (Rauf, 2014), torna-se preciso identificar as variáveis que podem interferir na sua ocorrência, pois este conhecimento pode ser útil nas estratégias organizacionais, assim como nas políticas e práticas de gestão de pessoas. Neste estudo pretende-se investigar o papel desempenhado pela percepção dos valores organizacionais enquanto núcleo da cultura organizacional e da confiança do empregado na organização em relação ao Civis- 
mo Organizacional. Esta expressão foi sugerida por Siqueira (1995) em substituição a Comportamentos de Cidadania Organizacional então considerada inadequada. Posteriormente, Porto e Tamayo $(2005$, p. 37) definiram o construto como "um comportamento organizacional que propicia a efetividade das organizações". Nota-se que na literatura internacional ainda predomina a expressão Cidadania Organizacional, embora em termos de aporte teórico e estudos empíricos, as denominações possam ser consideradas equivalentes.

Cavazotte et al. (2013), baseando-se em vários autores, acreditam que Comportamentos de Cidadania podem ser estimulados ou restringidos por fatores culturais, como por exemplo, o fato de em culturas coletivistas a manifestação de comportamentos altruístas ser mais provável que em culturas individualistas.

As perspectivas de investigação da cultura são amplas, dada a multiplicidade de suas facetas. Malbašić, Rey e Potočan (2014) destacam a importância dos valores na determinação dos comportamentos dos empregados, assim como na alocação de recursos. Para esses autores, os valores organizacionais são compartilhados e aceitos dentro das organizações.

Os valores organizacionais são aqueles percebidos pelos empregados como sendo efetivamente característicos da organização, o que, segundo Tamayo (2007), são princípios ou crenças compartilhadas pelos empregados que orientam o seu funcionamento e sua vida. Eles indicam as opções feitas pela organização por meio de sua história, sendo expressos por comportamentos, padrões de qualidade, estruturas organizacionais, estratégias de gerenciamento, dentre outros aspectos.

A confiança, segundo componente do modelo, é um atributo essencial nas relações estabelecidas entre grupos ou organizações que aspirem a preservar-se para além do presente, ou seja, faz parte de uma base em que se consolidam as relações interpessoais no seio de uma organização e as relações entre os grupos de uma organização (Siqueira \& Gomide, 2014). A confiança organizacional desempenha um papel crítico, que pode determinar a preservação ou a ruptura do rela- cionamento entre o empregado e a organização em que atua (Batista \& Oliveira, 2012; Nienaber, Hofeditz, \& Searle, 2014).

Chhetri (2014), em um ensaio teórico, supõe a ligação entre comportamentos de cidadania, justiça e confiança organizacionais explicando que a interdependência entre os membros de uma organização somente é possível quando há confiança entre eles. Assim, a confiança depositada pelo empregado em seus empregadores graças à possibilidade de se formularem políticas, pode impactar nos comportamentos de cidadania.

A definição de Confiança organizacional como um conjunto de crenças a respeito de padrões éticos da organização, de uso limitado de oportunismo em suas relações e de competência que se revela em sua capacidade técnica e solidez financeira (Ianaguivara, 2011), será adotada neste estudo cujo foco de análise recai nos aspectos da organização percebidos pelos empregados, não constituindo seu interesse a confiança na perspectiva das relações sociais.

Estudos já correlacionaram valores organizacionais e civismo (Jardim, 2009; Porto \& Tamayo, 2003, 2005); e valores organizacionais e confiança (Guardani, Teixeira, Bido, \& Mazzon, 2013; Oliveira, 2004; Oliveira \& Souza, 2014). No entanto, de acordo com a literatura, são escassos os estudos que tenham relacionado essas variáveis em um modelo integrado de investigação. Portanto, estudar o impacto dos valores organizacionais e da confiança do empregado na organização sobre os comportamentos de civismo significa incluir aspectos relativos à cultura organizacional e ao vínculo do empregado com sua organização de trabalho. Isso facilita o entendimento do processo de emissão de comportamento benéficos à organização que afetam não somente a relação do empregado com a organização mas também o desempenho dos indivíduos de que fazem parte (Guardani et al., 2013; Purohit, Patel, \& Purohit, 2014).

\section{Comportamentos de Civismo Organizacional}

A origem dos Comportamentos de Civismo Organizacional tem suas premissas no conceito 
de Cidadania Organizacional, que remete à perspectiva de Katz e Kahn (1974). Para estes autores é preciso que as organizações estimulem em seus membros o desejo de fazerem parte e de nelas permanecerem, ao mesmo tempo em que suas atitudes e resultados cumpram devidamente o que é esperado de cada um e contribuam com novas ideias e melhorias para as organizações. É postulado ainda que tal desejo precisa surgir, tanto por parte da organização quanto das pessoas.

Moorman (1991) também trouxe contribuições ao indicar que os atos de cidadania podem ser vistos como recursos de informações e de serviços. Nesse sentido, os comportamentos de cidadania seriam uma forma de retribuição social dos membros à organização caso nesta relação haja uma troca justa.

De acordo com Siqueira (1995), o termo cidadania organizacional recebeu críticas quanto a sua pertinência para designar os comportamentos representados. De fato, o termo Cidadania expressa, por um lado, a submissão à autoridade e por outro, o exercício do direito. Assim, a análise realizada pela autora sugere que tal conceito não se refere aos direitos dos trabalhadores, nem tampouco aos deveres que lhes seriam prescritos, mas sim a comportamentos executados pelos trabalhadores para a sua organização, embora não tenham obrigação de fazê-los (Siqueira, 1995). Portanto, parece inadequada a utilização deste termo no ambiente de trabalho para designar comportamentos espontâneos. Desse modo, como alternativa a autora sugeriu a expressão Civismo nas Organizações.

As contribuições de Siqueira (1995) tornaram-se alvo de estudo de Porto e Tamayo (2003), que construíram e validaram a Escala de Civismo nas Organizações, tendo como referencial o trabalho anterior da autora citada (Escala de Comportamento de Cidadania Organizacional).

Uma das contribuições deste estudo foi a definição de civismo organizacional: atos espontâneos dos trabalhadores que beneficiam o sistema organizacional, permitindo formas específicas de manifestação, embora não sejam previstas retribuições formais pela organização (Porto \& Tamayo, 2003). Além disso, contribuíram com a nova medida cujos índices de confiabilidade são superiores aos da Escala anterior, sendo os mesmos fatores confirmados empiricamente.

Já na literatura internacional o termo Cidadania Organizacional continua a ser utilizado como nas décadas anteriores, atraindo atenção de pesquisadores que investigam seus antecedentes e consequentes. Dentre as variáveis antecedentes, estudos recentes reforçam o papel da justiça organizacional (Asamani \& Mensah, 2013; Lee, Kim, \& Kim, 2013), e outros aspectos como qualidade de vida no trabalho (Kashani, 2012), efeito da personalidade, comprometimento organizacional, satisfação no trabalho e percepção de suporte organizacional sobre comportamentos de cidadania organizacional e sua implicação no desempenho (Hakim, Nimram, Haerani, \& Alam, 2014); e habilidade gerencial (Rapp, Bachrach, \& Rapp, 2013).

\section{Confiança nas Organizações}

A importância da confiança está associada aos vínculos estabelecidos pelo indivíduo com sua organização de trabalho, uma vez que os contratos e os controles formais não são os únicos meios existentes para se manter a tranquilidade das partes envolvidas nas relações dentro das organizações (Kramer, 1999; Oliveira \& Tamayo, 2008).

Embora tenha sua importância reconhecida, os teóricos alegam não existir consenso quanto à sua definição (Chhetri, 2014; Schoorman, Mayer, \& Davis, 2007). Isso se deve ao fato de o fenômeno ser investigado por várias áreas do conhecimento, o que leva a conceitos e a modelos diferentes. A confiança é abordada em alguns casos sob uma ótica, em outros como uma intenção ou também como uma variável da personalidade (Chhetri, 2014; Ferrin, Bligh, \& Kohles, 2007). Para Kramer (1999), a despeito das divergências existentes entre os vários teóricos, a confiança é fundamentalmente um estado psicológico na maioria das teorias sobre confiança.

Schoorman et al. (2007) a definem como "a vontade de uma pessoa de ser vulnerável às ações de outra parte com base na expectativa de que o outro irá executar uma ação específica" (p. 
712). Para Hosmer (1995) “Confiança é a expectativa de uma pessoa, grupo ou empresa de comportamentos eticamente justificáveis - isto é, decisões e ações moralmente corretas baseadas em princípios éticos de análise - por outra pessoa, grupo ou empresa numa diligência conjunta ou troca econômica" (p. 399).

Em relação à confiança organizacional (entre unidades ou entre organizações), encontra-se a definição proposta por Cummings e Bromiley (1996) o que a consideram como sendo:

a crença individual ou crença comum entre um grupo de pessoas em que outro indivíduo ou grupo: fazem esforço de boa fé para se comportarem de acordo com o compromisso firmado explícita ou implicitamente, são honestos em quaisquer negociações que precedam o compromisso além de não tomarem vantagem excessiva de outro quando a oportunidade ocorrer. (p. 303)

Considerando o nível organizacional, Costa (2000) afirma que a confiança refere-se à relação estabelecida com o sistema formal, sendo baseada em leis, regulamentos e nas práticas que mantem a organização como um todo.

Oliveira (2004), por sua vez, sugere uma definição para confiança alicerçada pela teoria cognitivista, ao propor o conceito que se refere a um conjunto de cognições interdependentes que integra crenças a respeito de padrões éticos, credibilidade da comunicação, poder econômico da organização e com capacidade de esta reconhecer o desempenho do empregado.

Esta definição elucida que a confiança existente na organização é formulada pelo indivíduo quando ele direciona seus esforços a fim de associar e processar as informações advindas deste contexto. Orientando-se nos mesmos princípios,

Ianaguivara (2011) propôs a seguinte definição: confiança organizacional refere-se a um conjunto de crenças a respeito de padrões éticos da organização, de uso limitado de oportunismo em suas relações e de competência que se revela em sua capacidade técnica e solidez financeira.

Nesse sentido, investigá-la na perspectiva do indivíduo em relação à sua organização de trabalho mostra-se útil à medida que novos modelos de gestão julgados seguros perdem sua es- tabilidade, levando à busca por outros caminhos possíveis que permitam o equilíbrio nas relações entre empregado e empregador (Zanini, Lusk, \& Wolff, 2009).

De acordo com Oliveira e Souza (2014), a produção científica sobre confiança no âmbito organizacional tem crescido nos últimos anos, o que pode ser ilustrado com alguns estudos que relacionam este construto a variáveis tais como gestão organizacional (Drummond, 2007; Novelli, Fischer, \& Mazzon, 2006; Reina \& Reina, 2007); comportamento cooperativo (Ferrin et al., 2007) e comprometimento organizacional (Chhetri, 2014; Song \& Kim, 2009); cidadania organizacional (Cavazotte et al., 2013; Chhetri, 2014; Hakim et al., 2014; Hassan \& Semerciöz, 2010); satisfação no trabalho (Hakim et al., 2014; Leat \& El-Kot, 2009); e valores pessoais e organizacionais (Oliveira \& Souza, 2014).

\section{Valores Organizacionais}

Segundo Jardim (2009), os estudos dos valores humanos tem interessado a diversas áreas do conhecimento. Em se tratando da cultura, os valores são centrais, pois viabilizam o aparecimento de comportamentos adaptativos aos diversos ambientes e aumentam a capacidade de enfrentamento do indivíduo (Jardim, 2009). De um modo geral, estudar valores implica compreender como eles estão vinculados e presentes nos indivíduos de diversas culturas, sendo necessários para analisar as ações do ser humano; uma vez que impactam o pensar, o sentir e o agir dos indivíduos (Tamayo, 2008).

Em conformidade com Silva e Zanelli (2014), na perspectiva etnográfica, a cultura se refere aos conhecimentos, crenças, arte, moral, leis, costumes ou outras capacidades ou hábitos adquiridos pelo homem como ser social. Já no âmbito organizacional, a compreensão dos processos organizacionais perpassa a questão da cultura. A importância atribuída por uma organização a certos valores pode determinar a quantidade de esforço investida por seus membros na emissão de certos comportamentos, bem como a persistência em sua execução (Tamayo, 2008). 
Para Tamayo e Gondim (1996), a função dos valores é orientar a vida da organização e guiar o comportamento dos seus membros, delimitando a forma de agir, pensar e julgar das pessoas. Da mesma forma, Jardim (2009) salienta que a função dos valores está em direcionar a vida da organização e em orientar os comportamentos de seus membros, uma vez que são determinantes da rotina diária na organização. Além do comportamento, os valores influenciam o julgamento que o sujeito faz do comportamento dos outros (Malbašić et al., 2014; Tamayo, 2008).

Oliveira e Tamayo (2004) ressaltam que os valores organizacionais atuam como mediadores nos conflitos contribuindo para a solução dos problemas da organização e desempenhando um papel importante no alcance dos objetivos organizacionais e no atendimento das necessidades dos indivíduos. O papel dos valores explicita a dimensão cultural que incentiva pesquisadores a entenderem a cultura organizacional e a modificarem-na para se adequar às exigências do meio organizacional (Oliveira \& Gomide, 2012).

No Brasil, estudos quantitativos sobre valores organizacionais são realizados com frequência (Estivalete, Andrade, Gomes, \& Costa, 2012; Ferreira \& Fernandes, 2009; Guardani et al., 2013; Jardim, 2009; Oliveira \& Souza, 2014; Porto \& Tamayo, 2005; Tamayo \& Borges, 2001). Na literatura internacional relaciona-se o estudo de Cavazotte et al. (2013), Malbašić et al. (2014), Moorman e Blakely (1995) e Spitzmüller, Van Dyne e Remus (2008).

\section{Método}

A amostra foi composta por 230 trabalhadores vinculados a organizações, dos quais $58,7 \%$ correspondem ao gênero feminino e $37,4 \%$ ao gênero masculino. O nível de escolaridade variou entre o ensino médio completo e a pós-graduação, predominando na amostra participantes com ensino superior incompleto $(75,7 \%)$ e vinculados a organizações privadas $(84,7 \%)$. A idade dos participantes variou de 18 a 49 anos com média igual a 27 anos $(D P=7,01)$ e o tempo médio de trabalho, de 3,2 anos $(D P=$ $3,77)$.
Os instrumentos de pesquisa utilizados na coleta de dados foram: (a) Inventário de Perfis de Valores Organizacionais - IPVO (Oliveira \& Tamayo, 2004) - composto pelos fatores Realização, Conformidade, Domínio, Tradição, Bem-estar do Empregado, Autonomia, Prestígio Organizacional e Preocupação com a Coletividade, cujos índices de precisão (Alpha de Cronbach) variam de 0,75 a 0,87 . Os itens foram avaliados em uma escala de seis pontos $(0=$ não se parece em nada com minha organização, 5 = é muito parecida com minha organização); (b) Inventário de Confiança do Empregado na Organização (Ianaguivara, 2011) - avalia os fatores Confiança na Ética, Confiança na Competência e Oportunismo, sendo que a confiabilidade variou de 0,84 a 0,96; (c) Escala de Civismo nas Organizações (Porto \& Tamayo, 2003), composta pelos fatores Sugestões Criativas ao Sistema, Proteção ao Sistema, Criação de Clima Favorável, Autotreinamento e Cooperação com colegas, cujos itens são avaliados em uma escala Likert de cinco pontos $(1=$ nunca; $5=$ sempre). O Alpha de Cronbach variou de 0,80 a 0,91. Observa-se que os valores de Alpha de Cronbach são superiores a 0,70 em todos os instrumentos, sendo avaliados como satisfatórios (Hair, Anderson, Tatham, \& Black, 2005).

As análises dos dados foram realizadas através do programa SPSS (Statistical Package of Social Science), versão 19. A análise exploratória dos dados foi efetuada com o objetivo de verificar a precisão da entrada de dados, respostas omissas e casos extremos, sendo realizados os ajustes necessários para os cálculos posteriores. A amostra foi descrita por meio de estatísticas descritivas (média, desvio padrão e frequência), enquanto as correlações entre as variáveis, verificadas através da correlação de Pearson. O teste do modelo, por sua vez, foi realizado mediante análise de regressão múltipla padrão para verificar as variáveis que se constituiriam as melhores preditoras dos comportamentos de civismo nas organizações.

A normalidade da distribuição foi testada pelo método Kolmogorov-Smirnov e apresentou significativa. Em seguida a análise dos histogramas com superposição da curva normal e dos 
índices de assimetria evidenciou diferenças de assimetria. Contudo, a grande maioria dos valores de assimetria permaneceu dentro de valores aceitáveis conforme parâmetros descritos por Miles e Shevlin (2001). Além da normalidade, foi testada a fidedignidade de cada um dos fatores dos instrumentos para a amostra deste estudo através do cálculo do Alpha de Cronbach, obtendo-se resultados próximos aos dos estudos originais, ou seja, valores superiores a 0,70 , indicando fidedignidade aceitável (Hair et al., 2005).

\section{Resultados e Discussão}

As correlações entre todas as variáveis foram significativas, em sua maioria positivas, apresentando somente uma negativa; com coeficientes variando de fracos $(r=0,11)$ a elevados $(r=0,75)$, de acordo com os critérios propostos por Dancey e Reidy (2006), segundo os quais correlações abaixo de 0,4 são fracas; correlações entre 0,4 a 0,49 - são moderadas e correlações acima de 0,5 são consideradas fortes.

A análise de Regressão Múltipla foi efetuada a fim de verificar quais as variáveis seriam as melhores preditoras dos comportamentos de civismo nas organizações, conforme pode ser visualizado na Tabela 1.

Os resultados da primeira análise de regressão (Tabela 1) mostram que os preditores significativos de Sugestões Criativas ao Sistema foram Confiança na Ética e o Valor Coletividade. A variância explicada no modelo foi igual a $23 \%, R^{2}$ ajustado $=0,19 ; F(10,195)=5,889 ; p<0,001$. Os coeficientes Beta indicam que confiança na ética é o preditor mais forte que o valor Coletividade na explicação de sugestões criativas, o que significa que a confiança nos princípios éticos adotados pelas organizações (honestidade, lealdade e transparência) tende a influenciar positivamente o empregado a oferecer sugestões criativas e a transmitir novas ideias para beneficiar a

\section{Tabela 1}

Resumo das Análises de Regressão Múltipla Padrão para as Variáveis Critério Relativas a Civismo Organizacional

\begin{tabular}{|c|c|c|c|c|c|c|}
\hline $\begin{array}{l}\text { Variável } \\
\text { Critério }\end{array}$ & Preditores & $B$ & $\beta$ & $\begin{array}{c}R \\
\text { múltiplo }\end{array}$ & $\begin{array}{c}R^{2} \\
\text { modelo }\end{array}$ & $\begin{array}{c}R^{2} \\
\text { ajustado }\end{array}$ \\
\hline \multirow[t]{2}{*}{ Sugestões criativas } & $\begin{array}{l}\text { Confiança } \\
\text { Ética }\end{array}$ & $0,52 * * *$ & $0,47 * * *$ & 0,47 & 0,22 & 0,18 \\
\hline & VO Coletividade & $-0,24 *$ & $-0,28 *$ & & & \\
\hline \multirow[t]{2}{*}{ Proteção ao sistema } & VO Conformidade & $0,22 * * *$ & $0,33 * * *$ & 0,56 & 0,31 & 0,27 \\
\hline & $\begin{array}{c}\text { Confiança } \\
\text { Competência }\end{array}$ & $0,19 *$ & $0,22 *$ & & & \\
\hline \multirow[t]{3}{*}{ Clima favorável } & VO Autonomia & $0,19^{*}$ & $0,24 *$ & 0,75 & 0,57 & 0,55 \\
\hline & $\begin{array}{c}\text { Confiança } \\
\text { Competência }\end{array}$ & $0,34 * *$ & $0,25 * *$ & & & \\
\hline & $\begin{array}{l}\text { Confiança } \\
\text { Ética }\end{array}$ & $0,37 * * *$ & $0,33 * * *$ & & & \\
\hline Autotreinamento & VO Autonomia & $0,37 * *$ & $0,40 * *$ & 0,52 & 0,27 & 0,23 \\
\hline Cooperação com colegas & VO Conformidade & $0,22 *$ & $0,20 *$ & 0,51 & 0,26 & 0,21 \\
\hline
\end{tabular}

Nota. $\mathrm{VO}=$ Valores Organizacionais.

$* p<0,05, * * p<0,01, * * * p<0,001$. 
organização. No entanto, quando os empregados percebem valores de bases éticas (Coletividade), a tendência é que haja uma diminuição na apresentação destas sugestões, o que teoricamente é inconsistente. Considerando a hipótese do fenômeno de supressão foi calculada outra análise de regressão eliminado o valor organizacional Coletividade. O resultado manteve Confiança na Ética como preditor significativo, $\beta=0,31$; $R^{2}=0,20 ; F(10,198)=4,925 ; p<0,001$, de Sugestões Criativas. Constatou-se por meio de outra análise de regressão que quando Confiança na Ética é eliminada do modelo, o valor Coletividade não apresenta contribuição significativa, revelando somente o valor Autonomia. Dessa forma, parece mais apropriado considerar como preditor de Sugestões Criativas a Confiança nos Componentes Éticos, assumindo a hipótese de supressão.

Não foram encontrados na literatura estudos que propusessem um modelo de investigação semelhante ao dessa pesquisa. Contudo, os resultados dos antecedentes de Civismo organizacional encontrados neste estudo se assemelham aos de Jardim (2009) que revelaram serem valores organizacionais preditores significativos dos comportamentos de civismo organizacional no Judiciário Mineiro.

Na segunda análise de regressão, para Proteção ao Sistema os preditores significativos foram os valores Conformidade e Coletividade e Confiança na Competência Organizacional ao explicarem $33 \%$ da variância do modelo, $R^{2}$ ajustado $=0,29 ; F(11,194)=8,650 ; p<0,001$. $\mathrm{O}$ coeficiente Beta indica que valor Conformidade é o preditor mais forte no caso de Proteção ao Sistema. No que diz respeito a regras e modelos de conduta a definição de limites das ações de seus membros influencia a emissão dos seguintes comportamentos: zelar e usar de forma adequada os equipamentos e cuidar da limpeza do ambiente de trabalho e contribuem também para a emissão destes comportamentos a confiança no saber fazer, propriedade da organização que possui conhecimento e que domina melhor que outras organizações as tecnologias em sua área de atuação. Em contrapartida, quando os empregados não percebem valores de bases éticas (Co- letividade) a tendência é que haja uma redução de ações voltadas para a proteção ao sistema.

Criação de Clima Favorável, terceira variável critério, apresentou os seguintes preditores significativos: valor Autonomia, Confiança na Competência e Ética organizacionais, $R^{2}$ ajustado $=0,55 ; F(11,194)=23,512 ; p<0,001$. A confiança nos princípios éticos adotados pelas organizações assim como em sua competência, tendem a influenciar positivamente o empregado a divulgar as vantagens e méritos da organização para outras pessoas externas ao ambiente de trabalho. As metas do valor Autonomia (curiosidade, criatividade, variedade de experiências) também contribuem para que o empregado defenda, elogie os produtos e serviços da organização. Assim, ao perceber este valor e confiar em sua organização, há o esforço do empregado em manter a imagem positiva dela no mercado, ao fornecer boas informações e apresentar as vantagens de nela trabalhar.

Porto e Tamayo (2005) também concluíram que para o fator Criação de Clima favorável no ambiente externo, os valores Domínio e Autonomia apresentaram-se como preditores significativos, mostrando que valores indicadores de metas organizacionais que estimulam a inovação e a criatividade fazem com que sejam emitidos mais comportamentos de criação de clima favorável à organização no ambiente externo.

Este resultado encontrado para clima favorável à organização se aproxima ao que Chiuzi (2006) apresentou em seu estudo ao averiguar as dimensões da organização positiva e seus impactos sobre o bem-estar dos trabalhadores, indicando como principal preditor a confiança do empregado na organização, cujos reflexos na prática referem-se a divulgação de uma imagem positiva da organização.

Os preditores significativos de Autotreinamento, quarta variável critério, foram os valores Autonomia e Coletividade que explicaram 26\% da variância, $R^{2}$ ajustado $=0,22 ; F(10,195)$ $=6,756 ; p<0,001$. Estes resultados indicam que à medida que o funcionário possui abertura para novos desafios, tende a buscar cursos e eventos que contribuam para seu desempenho no trabalho. Tais resultados convergem com a 
constatação de estudos que indicam comportamentos de cidadania organizacional e construtos relacionados, como por exemplo, o desempenho contextual que possuem relações significativas com os resultados organizacionais, tais como a produtividade ao nível da unidade, rentabilidade, eficiência e volume de negócios (por exemplo, Podsakoff, Whiting, Podsakoff, \& Blume, 2009; Podsakoff, Whiting, Podsakoff, \& Mishra, 2011).

Observa-se que valor Coletividade apresentou beta negativo e explicou que quando os empregados percebem valores de bases éticas, a tendência é que haja uma diminuição de comportamentos de autotreinamento por parte dos funcionários, verificando-se assim, inconsistência teórica no presente estudo. Diante da hipótese de supressão foi calculada outra análise de regressão eliminando o valor organizacional Coletividade, e o resultado mostrou que valor $\mathrm{Au}$ tonomia $(\beta=0,40)$ e confiança na Competência $(\beta=0,23)$, se mostraram preditores significativos de Autotreinamento, $R^{2}=0,23 ; F(9,196)=$ 6,$678 ; p<0,001$. Dessa forma, permaneceram como preditores o valor Autonomia e Confiança na Competência, indicando que se há percepção de abertura para novos desafios e confiança na competência da organização, o empregado tende a buscar meios de se atualizar que contribuam para seu desempenho no trabalho.

Porto e Tamayo (2005) obtiveram em seu estudo resultados semelhantes ao verificarem que os valores de Autonomia foram preditores significativos para o fator Autotreinamento. Tais comportamentos levam à inserção de inovações no trabalho por meio da aprendizagem de novas habilidades e conhecimentos, sendo coerentes com os valores que promovem a busca de novas soluções para os problemas organizacionais.

Ozer (2011), ao investigar a relação entre comportamentos de cidadania e desempenho no trabalho, verificou que autonomia é um preditor importante, pois aumenta a capacidade dos empregados para incorporar a ajuda que recebem de seus colegas em suas atividades, e este tipo de relacionamento influencia positivamente o desempenho no trabalho. Para esse autor, este resultado deve despertar o interesse das orga- nizações no sentido de promover um ambiente de trabalho social e psicológico favorável para a realização dos objetivos organizacionais através da criação de oportunidades para os funcionários desempenharem seus comportamentos de cidadania.

A Cooperação com os Colegas, última variável critério, teve sua variância explicada em $25 \%, R^{2}$ ajustado $=0,20 ; F(10,195)=6,321$; $p<0,001$, pelo valor Conformidade e Confiança na Competência da organização, indicando que valores relacionados à definição de limites das ações e a comportamentos dos membros da organização assim como a confiança no saber fazer da organização tendem a influenciar positivamente os empregados no sentido de auxiliar seus colegas de trabalho. Tais resultados sugerem que nas situações em que há limites das ações organizacionais e comportamentos bem estabelecidos, assim como capacidade de gerir adequadamente os conhecimentos e tecnologias existentes, o trabalhador tende a agir no sentido de cooperar, auxiliando os colegas de trabalho para beneficiar a organização. $\mathrm{O}$ sentido de cooperar é comparável à definição proposta por Organ, Podsakoff, e MacKenzie (2006) ao relatarem que o comportamento de ajuda refere-se a uma ajuda voluntária para com os outros, de modo a prevenir a ocorrência de problemas relacionados ao trabalho.

Os resultados deste estudo possuem semelhanças com estudos já citados, que encontraram correlações significativas entre Civismo e Valores, Civismo e Confiança, Valores e Confiança Organizacionais (Almeida \& Ferreira, 2010; Chiuzi, 2006; Guardani et al., 2013; Horta, Demo, \& Roure, 2012; Jardim, 2009; Ozer, 2011; Podsakoff et al., 2009; Podsakoff, et al., 2011). Ainda, este trabalho corrobora as informações contidas na literatura que apontam para o peso explicativo das variáveis Valores e Confiança Organizacionais na predição de Civismo Organizacional.

\section{Conclusões}

Este estudo teve como objetivo o teste de um modelo que buscou verificar se valores e 
confiança organizacionais são antecedentes dos comportamentos de civismo nas organizações, alcançando seu objetivo ao se confirmarem como preditores a confiança na Ética e na Competência organizacionais, bem como os valores Coletividade, Conformidade e Autonomia.

Identificou-se que confiança na ética da empresa foi preditor significativo de Sugestões Criativas ao Sistema e de Clima Favorável, ao mostrar que a confiança nos padrões éticos organizacionais tem reflexos na emissão de sugestões e de novas ideias para a organização, bem como na divulgação de seus benefícios para pessoas externas a ela. Por sua vez, Confiança na Competência foi preditor de Proteção ao Sistema, Clima Favorável, Autotreinamento e Cooperação com colegas. Isso mostra que diante de uma organização que "sabe fazer", que utiliza bem os conhecimentos e as tecnologias em sua área de atuação, o funcionário tende a zelar pelos equipamentos, utilizando-os de modo adequado, além de divulgar os benefícios existentes externamente, de buscar se aprimorar constantemente e de colaborar com os colegas nas diversas situações do trabalho.

Em relação aos Valores organizacionais, Conformidade foi preditor significativo de Proteção ao sistema e Cooperação com colegas, ao mostrar que quando há definição de limites das ações organizacionais e dos comportamentos de seus membros, com prioridade ao respeito a regras e modelos de comportamentos, o funcionário tende a zelar pelo patrimônio da organização e a ajudar colegas de trabalho por meio de conhecimentos, a auxiliar em problemas pessoais e contribuir na resolução de problemas no ambiente de trabalho.

O Valor Autonomia, por sua vez, explicou Clima Favorável e Autotreinamento, o que demonstra que o funcionário que procura o aperfeiçoamento constante e tem abertura para novos desafios, tende a defender, elogiar, descrever as qualidades da organização, transmitir a melhor das impressões para as pessoas e mostrar suas vantagens externamente, bem como procura frequentar cursos de reciclagem, e participar de eventos na área de atuação; enquanto que Valor
Coletividade foi preditor de Proteção ao sistema, ao mostrar que diante de valores de bases éticas, a tendência é zelar pelo patrimônio.

Os resultados mostram claramente que nem todos os valores organizacionais têm peso significativo na explicação de civismo organizacional assim como oportunismo, uma faceta avaliada da confiança que foi eliminada das análises. Do ponto de vista da cultura e gerencial, este dado leva a reflexão sobre quais valores podem estimular tais comportamentos. Além disso, a confiança é um aspecto central, pois vincula o empregado a organização, criando a base de uma relação de troca social, tão necessária ao funcionamento das organizações, além de propiciar vantagens aos empregados.

Ozer (2011) mostra que à medida que um funcionário expressa comportamentos de cidadania demonstra confiabilidade, o que gera gratidão e sentimentos de uma obrigação com os colegas de trabalho e a organização, no sentido de retribuir tais comportamentos. Por sua vez, essa relação amigável e de suporte promove a confiança, empatia e uma intenção de reciprocidade entre os colegas de trabalho (Hoppes \& Holley, 2014; Nienaber et al., 2014; Siqueira, 2001).

Por se tratar de um modelo de investigação recente sugere-se como agenda de pesquisas, a inclusão de outras variáveis como políticas de recursos humanos e variáveis atitudinais que possam ser mediadoras dos comportamentos de civismo. Ademais, há a necessidade de estudos com amostras variadas, pois no presente estudo foi utilizada uma amostra de conveniência específica de uma região do país e, consequentemente, não é conveniente generalizar os resultados.

Outras limitações encontradas neste estudo se referem à não utilização do Escalonamento Multidimensional, pois especificamente no caso de valores seria recomendável o uso desta técnica, o que poderia revelar aspectos a eles pertinentes; como também é necessário investigar as consequências do civismo nas organizações, haja vista sua importância.

Acredita-se, desse modo, que as contribuições deste trabalho possam ser utilizadas na gestão de pessoas, pois se a organização estimular 
comportamentos de civismo, poderá contar com empregados mais envolvidos no trabalho, interessados em cooperar nas atividades e com seus colegas, além da busca de aprimoramento e esforços visando o alcance de resultados organizacionais, por meio de suas competências.

\section{Referências}

Almeida, S. P., \& Ferreira, M. C. (2010). O impacto das atitudes frente às mudanças organizacionais nos comportamentos de cidadania. Psicologia: Ciência e Profissão, 30(3), 492-503. doi:10.1590/ S1414-98932010000300005

Asamani, A. L., \& Mensah, O. (2013). To what extent does employees perception of organizational justice influence their organizational citizenship behaviour? European Journal of Business and Management, 5(16), 32-41.

Bastos, A. V. B., Siqueira, M. M. M., \& Gomes, A. C. P. (2014). Cidadania organizacional. In M. M. M. Siqueira (Ed.), Novas medidas do comportamento organizacional: Ferramentas de diagnóstico e gestão (pp. 79-103). Porto Alegre, RS: Artmed.

Batista, L. R., \& Oliveira, A. F. (2012). Antecedentes da confiança do empregado na organização. Estudos de Psicologia (Natal), 17(2), 247-254. doi:10.1590/S1413-294X2012000200008

Bolino, M. C., Klotz, A. C., Turnley, W. H., \& Harvey, J. (2013). Exploring the dark side of organizational citizenship behavior. Journal of Organizational Behavior, 34(4), 542-559.

Cavazotte, F., Hartman, N. S., \& Bahiense, E. (2013). Charismatic leadership, citizenship behaviors, and power distance orientation: Comparing Brazilian and U.S. Workers. Cross-Cultural Research, 48(1), 3-31. doi:10.1177/1069397113494687

Chhetri, P. (2014). The role of cognitive and affective trust in the relationship between organizational justice and organizational citizenship behavior: A conceptual framework. Business: Theory and Practice, 15(2), 170-178. doi:10.3846/btp.2014.17

Chiuzi, R. M. (2006). As dimensões da organização positiva e seus impactos sobre bem-estar no trabalho (Dissertação de mestrado, Instituto de Psicologia da Universidade Metodista de São Paulo, São Bernardo do Campo, SP, Brasil).
Costa, A. C. (2000). A confiança nas organizações: Um imperativo nas práticas de gestão. In S. B. Rodrigues \& M. P. Cunha (Eds.), Estudos organizacionais: Novas perspectivas na administração de empresas: Uma coletânea luso-brasileira (pp. 284- 305). São Paulo, SP: Iglu.

Cummings, L. L., \& Bromiley, P. (1996). The organizational trust inventory. In R. M. Kramer \& T. R. Tyler (Eds.), Trust in organizations: Frontiers of theory and research (pp. 302-330). Thousand Oaks, CA: Sage.

Dancey, C. P., \& Reidy, J. (2006). Estatística sem matemática para psicologia. Porto Alegre, RS: Artmed.

Drummond, V. S. (2007). Confiança e liderança nas organizações. São Paulo, SP: Thomson Learning.

Estivalete, V. F. V., Andrade, T., Gomes, T. C., \& Costa, V. F. (2012). Valores organizacionais no Brasil: Uma análise da produção científica na área da administração nos últimos 10 anos. Gestão Contemporânea, 9(12), 43-68.

Ferreira, M. C., \& Fernandes, H. A. (2009). O impacto dos valores pessoais e organizacionais no comprometimento com a organização. Psico-USF, 14(3), 341-354. doi:10.1590/S141382712009000300010

Ferrin, D. L., Bligh, M. C., \& Kohles, J. C. (2007). Can I trust you to trust me? A theory of trust, monitoring, and cooperation in interpersonal and intergroup relationships. Group \& Organizational Management, 32(4), 465-499. doi: $10.1177 / 1059601106293960$

Guardani, F., Teixeira, M. L. M., Bido, D. S., \& Mazzon, J. A. (2013). A relação entre valores, práticas organizacionais e confiança de clientes no setor de serviços. Produção, 23(4), 806-817. doi:10.1590/S0103-65132013005000019

Hair, J. F., Anderson, R. E., Tatham, R. L., \& Black, W. C. (2005). Análise multivariada de dados. Porto Alegre, RS: Bookman.

Hakim, W., Nimram, U., Haerani, S., \& Alam, S. (2014). The antecedents of organizational citizenship behavior (OCB) and their effect on performance: Study on public university in Makassar, South Sulawesi, Indonesia. Journal of Business and Management, 16(2), 5-13.

Hassan, M., \& Semerciöz, F. (2010). Trust in personal and impersonal forms its antecedents and consequences: A conceptual analysis within or- 
ganizational context. International Journal of Management and Information Systems, 14(2), 67-83. doi:10.19030/ijmis.v14i2.832

Hoppes, C. R., \& Holley, K. A. (2014). Organizational trust in times of challenge: The impact on Faculty and Administrators. Innovation High Education, 9, 201-216. doi:10.1007/s10755013-9275-y

Horta, P., Demo, G., \& Roure, P. (2012). Políticas de Gestão de Pessoas, confiança e bem-estar no trabalho: Estudo em uma multinacional. Revista de Administração Contemporânea, 16(4), 566-585. doi:10.1590/S1415-65552012000400005

Hosmer, L. T. (1995). Trust: The connecting link between organizational theory and philosophic ethics. Academy of Management Review, 20(2), 379-403.

Ianaguivara, C. A. (2011). Confiança do empregado na organização: Revalidação de instrumento de medida (Dissertação de mestrado, Instituto de Psicologia, Universidade Federal de Uberlândia, MG, Brasil).

Jardim, M. F. (2009). Valores organizacionais e civismo no Sistema Judiciário Mineiro (Dissertação de mestrado, Faculdade Novos Horizontes, Belo Horizonte, MG, Brasil).

Kashani, F. H. (2012). A review on relationship between quality of work life and organizational citizenship behavior (Case Study: An Iranian Company). Journal of Basic and Applied Scientific Research, 2(9), 9523-9531.

Katz, D., \& Kahn, R. L. (1974). Psicologia social das organizações. São Paulo, SP: Atlas.

Kramer, R. M. (1999). Trust and distrust in organizations: Emerging perspectives, enduring questions. Annual Review of Psychology, 50(1), 569598. doi:10.1146/annurev.psych.50.1.569

Leat, M., \& El-Kot, G. (2009). Interpersonal trust at work, intrinsic motivation, work-related tension and satisfaction in Egypt. International Journal of Workplace Health Management, 2(2), 180194. doi:10.1108/17538350910970237

Lee, U. H., Kim, H. K., \& Kim, Y. H. (2013). Determinants of organizational citizenship behavior and its outcomes. Global Business \& Management Research: An International Journal, 5(1), 54-65.

Malbašić, I., Rey, C., \& Potočan, V. (2014). Balanced organizational values: From to practice. Jour- nal of Business Ethics. Retrieved from http:// link.springer.com.ezproxy.libraries.wright. edu:2048/article/10.1007/s10551-014-2238-0

Miles, J., \& Schevlin, M. (2001). Applying regression $\&$ correlation. A guide for students and researchers. London: Sage.

Moorman, R. H. (1991). Relationship between organizational justice and organizational citizenship behaviors: Do fairness perceptions influence employee citizenship? Journal of Applied Psychology, 76(6), 845-855.

Moorman, R. H., \& Blakely, G. L. (1995). Individualism-collectivism as an individual difference predictor of organizational citizenship behavior. Journal of Organizational Behavior, 16(2), 127 142. doi:10.1002/job.4030160204

Nienaber, A. M., Hofeditz, M., \& Searle, R. H. (2014). Do we bank on regulation or reputation? A meta-analysis and meta-regression of organizational trust in the financial services sector international. Journal of Bank Marketing, 32(5), 367-407. doi:10.1108/IJBM-12-2013-0146

Novelli, J. G., Fischer, R. M., \& Mazzon, J. A. (2006). Fatores de confiança interpessoal no ambiente de trabalho. Revista Administração, 41(4), 442452. doi:10.1590/S0080-21072006000400008

Oliveira, A. F. (2004). Confiança do empregado na organização: Impacto dos valores pessoais, organizacionais e da justiça organizacional (Tese de doutorado, Instituto de Psicologia, Universidade de Brasília, DF, Brasil).

Oliveira, A. F., \& Gomide, S., Jr. (2012). Valores, confiança do empregado e bem-estar subjetivo. In H. Mendonça (Ed.) Saúde e bem-estar no trabalho: Dimensões individuais e culturais (pp. 203-228). São Paulo, SP: Casa do Psicólogo.

Oliveira, A. F., \& Souza, M. A. (2014). Confiança do empregado na organização: $\mathrm{O}$ impacto dos valores pessoais e organizacionais. Revista Psicologia: Organizações e Trabalho, 14(2), 204-217.

Oliveira, A. F., \& Tamayo, A. (2004). Inventário de perfis de valores organizacionais. Revista de Administração da Universidade de São Paulo, 39(2), 129-140.

Oliveira, A. F., \& Tamayo, A. (2008). Confiança do empregado na organização. In M. M. M. Siqueira (Ed.), Medidas do comportamento organizacional: Ferramentas de diagnóstico e de gestão (pp. 97-109). Porto Alegre, RS: Artmed. 
Organ, D. W. (1990). The motivational basis of organizational citizenship behaviour. Research in Organizational Behaviour, 12, 43-72.

Organ, D. W., Podsakoff, P. M., \& MacKenzie, S. B. (2006). Organizational citizenship behavior: Its nature, antecedents, and consequences. London: Sage.

Ozer, M. (2011). A moderated mediation model of the relationship between organizational citizenship behaviors and job performance. Journal of Applied Psychology, 96(6), 1328-1336. doi:10.1037/a0023644

Podsakoff, N. P., Whiting, S. W., Podsakoff, P. M., \& Blume, B. D. (2009). Individual- and organizational-level consequences of organizational citizenship behaviors: A meta-analysis. Journal of Applied Psychology, 94(1), 122-141. doi:10.1037/a0013079

Podsakoff, N. P., Whiting, S. W., Podsakoff, P. M., \& Mishra, P. (2011). Effects of organizational citizenship behaviors on selection decisions in employment interviews. Journal of Applied Psychology, 96(2), 310-326. doi:10.1037/a0020948

Porto, J. B., \& Tamayo, A. (2003). Desenvolvimento e validação da Escala de Civismo nas Organizações. Estudos de Psicologia (Natal), 8(3), 393402. doi:10.1590/S1413-294X2003000300006

Porto, J. B., \& Tamayo, A. (2005). Valores organizacionais e civismo nas organizações. Revista de Administração Contemporânea, 9(1), 35-52. doi:10.1590/S1415-65552005000100003

Purohit, B., Patel, D., \& Purohit, S. (2014). A study of organizational values in government run primary health centres in India. Journal of Health Management, 16(2), 303-313. doi:10.1177/0972063414526119

Rapp, A. A., Bachrach, D. G., \& Rapp, T. L. (2013). The influence of time management skill on the curvilinear relationship between organizational citizenship behavior and task performance. Journal of Applied Psychology, 98(4), 668-677. doi:10.1037/a0031733

Rauf, F. H. A. (2014). Perception of organizational justice as a predictor of organizational citizenship behavior: An empirical study at schools in Sri Lanka. European Journal of Business and Management, 6(12), 124-130.

Reina, D., \& Reina, M. (2007). Building sustainable trust. OD Practitioner, 39(1), 36-41.
Schoorman, F. D., Mayer, R. C., \& Davis, J. H. (2007). An integrative model of organizational trust: Past, present and future. Academy of $\mathrm{Ma}$ nagement Review, 32(2), 344-354. doi:10.5465/ AMR.2007.24348410

Silva, N., \& Zanelli, J. C. (2014). Cultura organizacional. In J. C. Zanelli, J. E. Borges-Andrade, \& A. V. B. Bastos (Eds.), Psicologia, organizações e trabalho no Brasil (pp. 491-546). Porto Alegre, RS: Artmed.

Siqueira, M. M. M. (1995). Antecedentes do comportamento de cidadania organizacional: A análise de um modelo pós-cognitivo (Tese de doutorado, Instituto de Psicologia, Universidade de Brasília, DF, Brasil).

Siqueira, M. M. M. (2001). Comportamentos de cidadania organizacional. In M. M. M. Siqueira, S. Gomide Jr., \& A. F. Oliveira (Eds.), Cidadania, justiça e cultura nas organizações: Estudos psicossociais (pp. 22-52). São Bernardo do Campo, SP: Editora da Universidade Metodista de São Paulo.

Siqueira, M. M. M., \& Gomide, S., Jr. (2014). Vínculos do indivíduo com o trabalho e com a organização. In J. C. Zanelli, J. E. BorgesAndrade, \& A. V. B. Bastos (Eds.), Psicologia, organizações e trabalho no Brasil (pp. 316328). Porto Alegre, RS: Artmed.

Song, J. H., \& Kim, H. M. (2009). The integrative structure of employee commitment: The influential relations of individuals' characteristics in a supportive learning culture. Leadership \& Organizational Development Journal, 30(3), 240255. doi:10.1108/01437730910949526

Spitzmüller, M., Van Dyne, L., \& Remus, I. (2008). Organizational citizenship behavior: A review and extension of its nomological network. In J. Barling \& C. L. Cooper, The Sage Handbook of Organizational Behavior: Vol. 1. Micro approaches (pp. 106-123). London: Sage.

Tamayo, A. (2007). Contribuições ao estudo dos valores pessoais, laborais e organizacionais [Número especial]. Psicologia: Teoria e Pesquisa, 23, 1724. doi:10.1590/S0102-37722007000500004

Tamayo, A. (2008). Valores organizacionais. In M. M. M. Siqueira (Ed.), Medidas do comportamento organizacional: Ferramentas de diagnóstico e de gestão (pp. 309-340). Porto Alegre, RS: Artmed. 
Tamayo, A., \& Borges, L. O. (2001). Valores del trabajo y valores de las organizaciones. In $\mathrm{M}$. Ros \& V. V. Gouveia (Eds.), Psicología de los valores humanos: desarollos teóricos, metodológicos y aplicados (pp. 325-352). Madrid: Biblioteca Nueva.

Tamayo, A., \& Gondim, M. G. C. (1996). Escala de valores organizacionais. Revista de Administração da Universidade de São Paulo, 31(2), 62-72.

Zanini, M. T., Lusk, E., \& Wolff, B. (2009). Confiança dentro das organizações da nova economia:
Uma análise empírica sobre as consequências da incerteza institucional. Revista Administração Contemporânea, 13(1), 72-91. doi:10.1590/ S1415-65552009000100006

Recebido: 03/02/2015

$1^{a}$ revisão: $31 / 07 / 2015$

$2^{a}$ revisão: 03/09/2015

Aceite final: 10/09/2015 